\title{
Treatment of Fibromyalgia with Formula Acupuncture: Investigation of Needle Placement, Needle Stimulation, and Treatment Frequency
}

\author{
RICHARD E. HARRIS, Ph.D., ${ }^{1}$ XIAOMING TIAN, M.D., L.Ac., ${ }^{2}$ DAVID A. WILLIAMS, Ph.D., ${ }^{1}$ \\ THOMAS X. TIAN, C.M.D., L.Ac., ${ }^{2}$ THOMAS R. CUPPS, M.D. ${ }^{3}$ FRANK PETZKE, M.D., ${ }^{4}$ \\ KIMBERLY H. GRONER, M.S.N., ${ }^{1}$ PINAKI BISWAS, M.Stat. ${ }^{5}$ RICHARD H. GRACELY, Ph.D., ${ }^{1}$ \\ and DANIEL J. CLAUW, M.D. ${ }^{1}$
}

\begin{abstract}
Objectives: The objective of this study was to investigate whether typical acupuncture methods such as needle placement, needle stimulation, and treatment frequency were important factors in fibromyalgia symptom improvement.

Design/settings/subjects: A single-site, single-blind, randomized trial of 114 participants diagnosed with fibromyalgia for at least 1 year was performed.

Intervention: Participants were randomized to one of four treatment groups: (1) T/S needles placed in traditional sites with manual needle stimulation $(n=29)$ : (2) T/0 traditional needle location without stimulation $(n=30)$; (3) N/S needles inserted in nontraditional locations that were not thought to be acupuncture sites, with stimulation ( $n=28)$; and (4) N/0 nontraditional needle location without stimulation $(n=27)$. All groups received treatment once weekly, followed by twice weekly, and finally three times weekly, for a total of 18 treatments. Each increase in frequency was separated by a 2-week washout period.

Outcome measures: Pain was assessed by a numerical rating scale, fatigue by the Multi-dimensional Fatigue Inventory, and physical function by the Short Form-36.

Results: Overall pain improvement was noted with $25 \%-35 \%$ of subjects having a clinically significant decrease in pain; however this was not dependent upon "correct" needle stimulation $(t=1.03 ; p=0.307)$ or location $(t=0.76 ; p=0.450)$. An overall dose effect of treatment was observed, with three sessions weekly providing more analgesia than sessions once weekly $(t=2.10 ; p=0.039)$. Among treatment responders, improvements in pain, fatigue, and physical function were highly codependent (all $p \leq 0.002$ ).
\end{abstract}

Conclusions: Although needle insertion led to analgesia and improvement in other somatic symptoms, correct needle location and stimulation were not crucial.

\section{INTRODUCTION}

A cupuncture has been used as a therapeutic intervention for more than 2500 years in China, and remains an im- portant facet of many modern Asian medical systems. ${ }^{1}$ The use of acupuncture as a complementary and alternative form of medicine in Western countries has increased rapidly over the last three decades. ${ }^{2,3}$ The U.S. Food and Drug Admin-

\footnotetext{
${ }^{1}$ Department of Internal Medicine, Division of Rheumatology, University of Michigan, Ann Arbor, MI.

${ }^{2}$ Academy of Acupuncture and Chinese Medicine, Bethesda, MD.

${ }^{3}$ Department of Rheumatology, Georgetown University, Washington, DC.

${ }^{4}$ Department of Anesthesiology, University of Cologne, Cologne, Germany.

${ }^{5}$ Department of Biostatistics, University of Michigan, Ann Arbor, MI.
} 
istration (FDA) estimates that Americans spend half a billion dollars per year on acupuncture treatments, most commonly for conditions involving pain, ${ }^{4}$ despite the lack of agreement within the scientific community on its efficacy and mechanism of action. Of note, the most appropriate control intervention to use is undecided ${ }^{5}$ and it is not clear whether acupuncture efficacy is synergistically dependent on where the needles are placed and whether they are stimulated. ${ }^{6}$ As a control some investigators use nontraditional sites, ${ }^{7-9}$ whereas others insert needles in traditional acupuncture points but refrain from stimulating them. ${ }^{10,11}$

Fibromyalgia (FM), a condition characterized by chronic diffuse pain, is the second most common rheumatologic disorder, affecting $2 \%-4 \%$ of the populations of industrialized countries. ${ }^{12,13}$ To meet American College of Rheumatology criteria for this diagnosis, individuals must have a history of chronic pain in all four quadrants of the body plus the axial skeleton, and have 11 or more (of a possible 18) tender points. ${ }^{14}$ Although FM is partly defined by tenderness at these specific anatomic sites, in fact this condition is characterized by generalized tenderness, or hyperalgesia. ${ }^{15,16}$ As no universally effective, well-tolerated treatment exists, patients with FM often seek acupuncture. ${ }^{17}$ Yet, most studies on the efficacy of acupuncture in FM have been uncontrolled case series, ${ }^{18,19}$ and only a single high-quality controlled trial of 70 patients randomized to electro-acupuncture or sham electrical stimulation has provided evidence supporting acupuncture for this condition. ${ }^{11,20}$ Although seven of eight outcomes improved in the active treatment group compared to none in the sham group, the extent to which the sham group was blinded was not assessed.

To explore methodological acupuncture issues, a $2 \times 2$ factorial was used design to address the following questions: (1) Is acupuncture beneficial for FM? (2) Is the placement of needles in traditional locations, and stimulation of the needles, required for symptom improvement? and (3) Is there a dependence on treatment frequency? It was hypothesized that if needle location and/or stimulation were essential, then an additive or synergistic effect should be detected with traditional or correct needle placement and stimulation. In addition, if acupuncture produces analgesia via a physiologic mechanism, a frequency-dependent improvement in symptoms might be observed.

\section{METHODS}

\section{Recruitment}

Participants were recruited from the Washington, DC, metropolitan area from August 2000 through January 2002 via announcements in local newspapers and periodicals and were screened on the telephone for study eligibility. Inclusion criteria were: (1) having met ACR criteria for the diagnosis of $\mathrm{FM}^{14}$ for at least 1 year; (2) reported widespread pain on more than $50 \%$ of days; (3) willingness to limit the introduction of new medications or treatment modalities for FM symptoms. Exclusion criteria were: (1) sufficient knowledge of acupuncture techniques to prevent blinding (including previous acupuncture); (2) known bleeding diathesis; (3) autoimmune or inflammatory disease; (4) regular use of narcotic analgesics daily or a history of substance abuse; (5) contraindication to the use of acetaminophen or ibuprophen (rescue analgesics) ; (6) participation in other therapeutic trials; (7) pregnancy or lactation; or (8) receiving disability payments or were involved in litigation related to FM. Subjects were allowed to continue their normal treatment regime(s), including use of antidepressants); however they were asked not to make any additional changes and not to seek acupuncture outside of the trial.

All participants were randomized and allocated to one of four treatment arms using computer-generated random numbers in a four-block design. Treatment allocation was concealed in an opaque envelope and then presented to the acupuncturist 1 day before treatment. All procedures were approved by the local institutional review board; participants received full descriptions of the protocol and gave written informed consent.

\section{Treatment}

This study used a $2 \times 2$ factorial design to examine the individual and synergistic effects of both needle location and needle stimulation on the efficacy of acupuncture analgesia. The four intervention arms were: (1) T/S (traditional site with stimulation): subjects received acupuncture, at points lying on Traditional Chinese Medicine acupuncture meridians, combined with manual stimulation of the needles; (2) T/0 (traditional site without stimulation): participants received the needles placed at the traditional locations but without any needle manipulation, (3) N/S (nontraditional site with stimulation): participants received the same number of needles placed at the same depths and with the same degree of manual stimulation as the T/S group, but these needles were placed in sites that were not believed to be effective in Traditional Chinese Medicine-based acupuncture; and (4) N/0 (nontraditional site with no stimulation): subjects received needles in nontraditional sites without stimulation. Subjects were told that they would be randomly assigned to one of these treatment groups and would receive either traditional acupuncture therapy or control acupuncture techniques not known to benefit FM. The T/S arm represented traditional Chinese acupuncture whereas the other three arms could be considered as different control groups.

The active point formula (Du 20, LI 11, LI 4, GB 34, bilateral St 36, Sp 6, Liv 3, and Ear-Shenmen) ${ }^{1}$ was chosen based on the points' ability to relieve FM symptoms in Traditional Chinese Medicine. Needles placed in the nontraditional groups were not on acupuncture meridians or points (Fig. 1A). The choice of point locations was determined by 


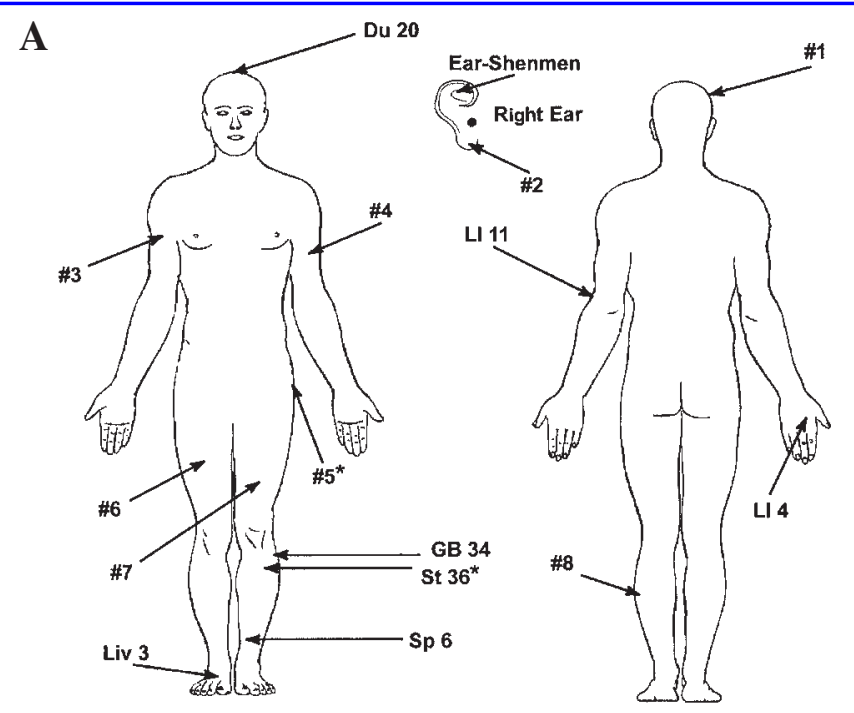

B

\begin{tabular}{|c|c|c|c|c|c|c|c|c|c|c|c|c|c|}
\hline Week & 0 & 1 & 2 & 3 & $4-5$ & 6 & 7 & 8 & 9-10 & 11 & 12 & 13 & 14-15 \\
\hline $\begin{array}{l}\text { Acupuncture sessions } \\
\text { per week }\end{array}$ & 0 & 1 & 1 & 1 & 0 & 2 & 2 & 2 & 0 & 3 & 3 & 3 & 0 \\
\hline Outcome Measures: & & & & & & & & & & & & & \\
\hline Pain (NRS) & $x$ & & & $x$ & $x$ & & & $x$ & $x$ & & & $x$ & $\mathrm{x}$ \\
\hline Fatigue (MFI) & $x$ & & & & $x$ & & & & $x$ & & & & $x$ \\
\hline Function (SF-36) & $x$ & & & & $x$ & & & & $x$ & & & & $\mathrm{x}$ \\
\hline
\end{tabular}

FIG. 1. Needle locations and temporal outline of study outcomes. A. Body maps of approximate needle locations. \#, nontraditional points; *, inserted bilaterally. B. Representation of study outcomes and acupuncture sessions by week. NRS, numeric rating scale; MFI, Multi-dimensional Fatigue Inventory; SF-36, Short Form-36.

licensed acupuncturists (X.T. and T.X.T., who each have 12 years of experience in treating FM and 17 years of experience with this form of acupuncture).

Treatments followed a forced-titration paradigm: once weekly for 3 weeks, then twice weekly for 3 weeks, and finally 3 times weekly for 3 weeks for a total of 18 sessions. A 2-week wash-out period was inserted between each treatment dose (Fig. 1B). Sterile, 2.54-cm, single-use, disposable, 38-gauge stainless steel needles were used (HBW Supply Inc., Hemet, CA). Stimulation was performed manually by lifting and thrusting while evenly rotating the needle ( $\sim 12$ times at 180 degrees clockwise and counterclockwise). Characteristic de qi needling sensations ${ }^{1}$ resulting from needle manipulation were elicited in the T/S group but not in the N/S group. There was no intention from the acupuncturist to elicit needle sensations in the N/S group. De qi was assessed by the acupuncturist's sensation of needle grasping and by the subject's report to a research assistant. Needles were retained for 20 minutes. More than $95 \%$ of the acupuncture sessions were performed by X.T., and the re- maining sessions were performed by licensed acupuncturists trained for this study.

\section{Blinding}

To ensure and to verify adequate blinding throughout the study multiple measures were taken. Participants from different treatment arms were never present in the acupuncture clinic at the same time. Subjects were asked to refrain from seeking additional information on acupuncture and from discussing acupuncture with other participants, during the time in the study. All subjects were blindfolded during treatment and no verbal communication was allowed between the acupuncturist and the subjects. A nonblinded research assistant was present during all sessions to monitor and ensure treatment integrity. This study was single-blinded. The acupuncturist knew the treatment groups and study hypothesis; however all evaluators of study outcomes were blinded to treatment allocation. Blinding was assessed by questionnaire following the third acupuncture session. This questionnaire read: "What type of procedure do you think you are receiving, acupuncture, placebo (sham), or can't tell."

\section{A priori outcomes}

Pain (primary outcome). Subjects completed a 101-point numeric rating scale (NRS) which ranged from 0 to 100 points in 5-point increments. The scale was anchored at 0 by "no pain" and 100 by "worst pain imaginable." Clinically meaningful treatment responders were determined using the criteria suggested by Farrar et al. ${ }^{21}$ : either a 20-point reduction or a $30 \%$ improvement in pain from baseline. On average $85.5 \%$ of responders met both criteria, while $9.8 \%$ met the $30 \%$ improvement criterion only and $4.6 \%$ met the 20-point reduction criterion only.

Fatigue. Participants completed the Multi-Dimensional Fatigue Inventory questionnaire ${ }^{22}$ and clinically meaningful changes in fatigue were calculated using the Reliability of Change Index (RC). ${ }^{23} \mathrm{~A}$ change of 3.67 in the General Fatigue score from baseline was used to classify fatigue responders. The General Fatigue scores range from 4 to 20 with larger scores indicating more fatigue.

Function. Physical function was assessed with the physical components score calculated from the Short Form-36 questionnaire. ${ }^{24}$ This score ranges from 0 to 100 with higher scores indicating better function. Previous work has suggested that a change of seven points in the physical components score is clinically meaningful and therefore we used this value as a cutoff for function responders.

\section{Analysis}

A priori it was estimated that a sample size of 30 patients per group was needed to detect $30 \%$ differences between 
groups with a power of 0.82 and a significance level of 0.05 . Longitudinal data were analyzed according to intention to treat. Incomplete data were handled via a last observation carried forward in time. Data were either hand entered or scanned via Teleform (Cardiff Software Inc., Bozeman, MT) and double-checked. Analysis was carried out using SAS (Cary, NC) and SPSS (Chicago, IL) software.

Analyses of variance were performed to determine differences in age, years of disease diagnosis, number of treatments attended, and baseline pain; $\chi^{2}$ tables were constructed for responder status, male/female ratio, and dropouts. Paired $t$-tests were performed to determine significant changes in NRS pain scores between weeks 3 and 4, 8 and 9 , and 13 and 15 .

Primary analyses contained two strategies: (1) assessment of changes in mean scores and (2) assessment of changes in binary responder classification. These two approaches were taken to examine the robustness of findings. To examine the effects of location, stimulation, and treatment frequency (dose) on pain, a repeated-measures model was fit to the data by calculating the change in response from baseline to weeks 3,8 , and 13 , including fixed effects for a combination of dose, location, and stimulus. Model 1 appeared as follows:

$$
y_{i j k l}-x_{l}=\mu+\alpha_{i j k}+\varepsilon_{i j k l} \quad \text { (Equation 1) }
$$

where $\alpha_{i j k}, i=1,2,3 ; j=1,2$ and $k=1,2$ represents the effect for the $i^{\text {th }}$ dose level, $j^{\text {th }}$ stimulus, and $k^{\text {th }}$ location; $x_{l}$ is the baseline response for the $t^{\text {th }}$ patient. A general correlation structure was assumed across the dose levels to account for within-patient variability across time, as time and dose were confounded in the experiment.

To analyze data from the binary pain outcome (responders versus nonresponders), a similar repeated-measures model was used (model 2). This model used a binomial assumption for the distribution and a logit link leading to a generalized linear model (GLM), as follows:

$$
\operatorname{logit} p_{i j k}=\mu+\alpha_{i j k} \quad \text { (Equation 2) }
$$

where $p_{i j k}$ represents the probability of responding. The other terms have the same interpretation as model 1 . Here, a response value of 1 indicates a 20-point or 30\% change on the NRS pain scale of the current pain rating compared to baseline.

\section{RESULTS}

\section{Baseline demographics and dropouts}

Subject demographics are displayed in Table 1. There were no significant group differences in duration of fibromyalgia $(F=0.070 ; p=0.976)$ or age $(F=2.186 ; p=$ $0.094)$. Not unexpectedly, the majority of subjects were female (106/114); however the ratio of female to male subjects was not significantly different across groups $\left(\chi^{2}=\right.$ $5.019, p=0.136)$. No differences were detected in pain scores between groups $(F=0.215 ; p=0.886)$.

Figure 2 depicts the flow of participants through the study. Thirty-three percent $(33 \% ; 38 / 114)$ of subjects dropped out of the study before completion. The majority of dropouts occurred in the first 4 weeks $(21 / 38 ; 55 \%)$; the primary reason for dropping out was the time commitment $(11 / 38)$. There were no significant group differences in dropout rates $\left(\chi^{2}=3.039, p=0.386\right)$, and the total number of treatment sessions attended did not vary by group $(F=$ 0.122; $p=0.947$; Table 1).

\section{Blinding assessment}

To assess whether subjects could determine to which treatment arm they had been randomized, they were asked in week 4 , if they thought they were receiving either (a) acupuncture or (b) placebo or (c) could not tell. A $\chi^{2}$ analysis revealed no significant differences (Fisher's exact $\chi^{2}=$ 7.531, $p=0.259$ ), indicating that participants remained blinded to treatment assignment at week 4 .

Table 1. Sociodemographic Characteristics of Fibromyalgia (FM)

\begin{tabular}{|c|c|c|c|c|c|}
\hline Parameter & $T / S$ & $T / 0$ & $N / S$ & $N / O$ & $\mathrm{p}$ \\
\hline$n$ & 29 & 30 & 28 & 27 & \\
\hline Age, years* & $46.0(10.1)$ & $44.5(10.9)$ & $51.3(10.0)$ & $48.1(10.9)$ & 0.094 \\
\hline Female & 29 & 27 & 24 & 26 & 0.136 \\
\hline Years of FM diagnosis* & $5.50(3.71)$ & $5.26(4.83)$ & $5.17(4.24)$ & $5.77(4.10)$ & 0.976 \\
\hline Treatments* & $15.7(1.89)$ & $15.6(1.80)$ & 15.9 (1.37) & $15.7(1.80)$ & 0.947 \\
\hline \multicolumn{6}{|l|}{ Ethnicity } \\
\hline White & 25 & 27 & 25 & 23 & \\
\hline African American & 2 & 2 & 2 & 4 & \\
\hline Hispanic & 0 & 0 & 0 & 0 & \\
\hline Other & 2 & 1 & 1 & 0 & \\
\hline
\end{tabular}
Study Participants and Average Number of Treatments

$\mathrm{T} / \mathrm{S}$, traditional needle locations with manual stimulation; T/0, traditional needle location without manual stimulation; N/S, nontraditional needle location with manual stimulation; N/0, nontraditional needle location without manual stimulation.

*Values are mean (standard deviation) by treatment group. 


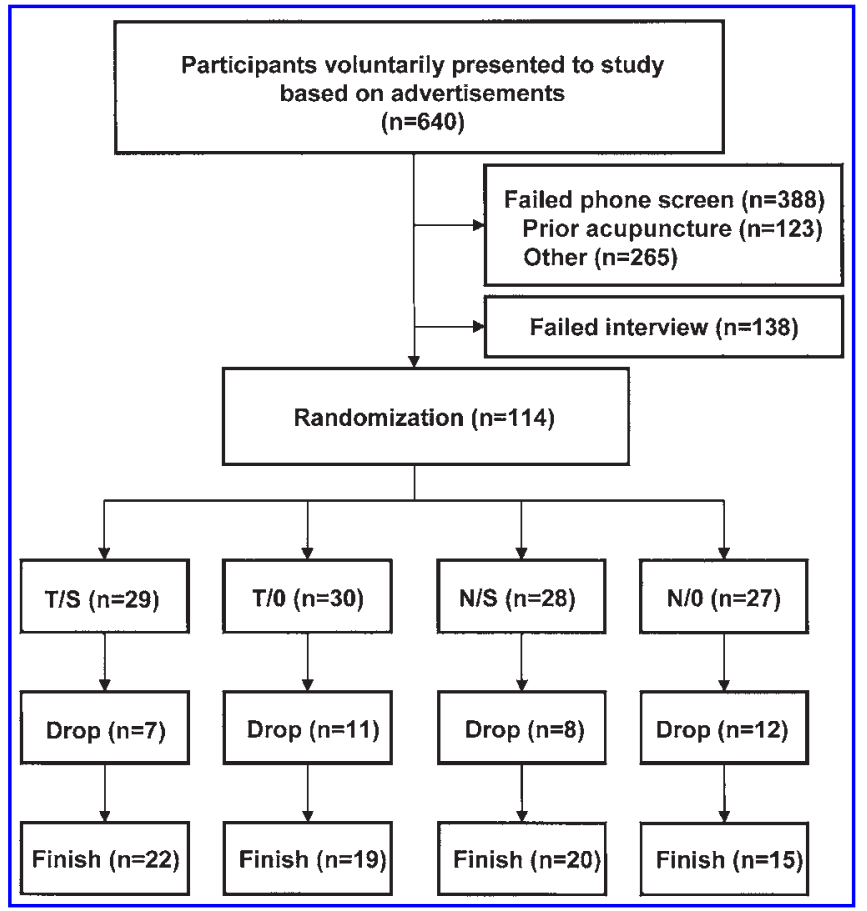

FIG. 2. Flow of participants through study. N/S, nontraditional needle location; T/S, traditional needle location; T/0, traditional needle location without stimulation; N/0, nontraditional needle without manual stimulation.

\section{Needle location and stimulation}

Mean pain, fatigue, and function scores are shown in Table 2 and responders are displayed in Table 3. Based on analysis using model 1 , no significant effects were obtained for either needle stimulation (weeks 3, 8, 13: $t=1.03 ; p=$ 0.307 ) or location (weeks 3, 8, 13: $t=0.76 ; p=0.450$ ). Similarly no significant effects were obtained from model 2 for the binary response pain variable for either needle stimulation (weeks 3, 8, 13: $\chi^{2}=3.60 ; p=0.058$ ) or location (weeks 3, 8, 13: $\chi^{2}=0.20 ; p=0.657$ ).

Figure 3A shows a graphic representation of the percentage of pain responders by time. No group consistently had more improvement than another. Taken together these results suggested that there was no additional benefit derived from placing needles in the correct location, or stimulating the needles, at these time points.

\section{Treatment frequency (cumulative dose)}

As exploratory analyses we tested for effects of treatment frequency in data from all four groups combined. A fit of model 1 to the continuous NRS pain scores yielded a significant overall effect for treatment frequency between weeks 4 and $15(t=4.81 ; p=0.045)$ and between weeks 3 and $13(t=4.92 ; p=0.039)$. No significant effects were

Table 2. Scores for Pain, Fatigue, and Function by Acupuncture Group and Week

\begin{tabular}{|c|c|c|c|c|c|}
\hline & $T / S$ & $T / 0$ & $N / S$ & $N / 0$ & Total \\
\hline \multicolumn{6}{|l|}{ Pain } \\
\hline Week 0 & $56.46(20.46)$ & $53.08(25.18)$ & 54.57 (24.77) & $58.33(19.70)$ & $55.38(22.59)$ \\
\hline Week 3 & $54.13(23.44)$ & $53.27(23.53)$ & $56.00(26.29)$ & $54.41(24.68)$ & $54.36(23.98)$ \\
\hline Week 4 & $62.95(22.55)$ & $55.80(27.07)$ & $60.45(26.41)$ & $60.29(25.59)$ & $59.77(25.15)$ \\
\hline Week 8 & $55.42(26.82)$ & $46.74(26.74)$ & $42.86(28.00)$ & $54.41(27.61)$ & $49.76(27.28)$ \\
\hline Week 9 & $48.26(28.59)$ & $51.92(27.02)$ & $46.82(30.69)$ & $60.83(22.31)$ & $51.52(27.55)$ \\
\hline Week 13 & $53.26(32.00)$ & $40.83(29.92)$ & $44.09(25.94)$ & $53.82(23.88)$ & $47.56(28.52)$ \\
\hline Week 15 & $54.17(32.09)$ & $53.85(28.79)$ & $46.30(25.50)$ & $56.11(19.14)$ & $52.47(27.12)$ \\
\hline Change $^{\mathrm{a}}$ & $3.20(26.23)$ & $12.25(27.55)$ & $10.48(25.36)$ & $4.51(21.79)$ & $7.82(25.56)$ \\
\hline$p$, location ${ }^{\mathrm{b}}$ & 0.450 & & & & \\
\hline$p$, stimulation $^{\mathrm{c}}$ & 0.307 & & & & \\
\hline \multicolumn{6}{|l|}{ Fatigue } \\
\hline Week 0 & $16.78(4.00)$ & $16.33(3.13)$ & $16.35(2.95)$ & $17.12(2.50)$ & $16.60(3.19)$ \\
\hline Week 4 & $16.35(3.65)$ & $15.68(3.46)$ & $16.26(3.39)$ & $15.78(3.46)$ & $16.02(3.44)$ \\
\hline Week 9 & $15.13(4.01)$ & $15.74(3.57)$ & $14.73(3.30)$ & $15.72(3.08)$ & $15.33(3.50)$ \\
\hline Week 15 & $15.71(3.59)$ & $14.59(4.55)$ & $14.35(4.20)$ & $15.39(2.75)$ & $14.98(3.89)$ \\
\hline Change $^{\mathrm{a}}$ & $1.08(2.95)$ & $1.74(3.22)$ & $2.00(3.28)$ & $1.73(2.95)$ & $1.62(3.04)$ \\
\hline \multicolumn{6}{|l|}{ Function } \\
\hline Week 0 & $31.80(7.35)$ & $37.60(9.22)$ & $37.20(8.70)$ & $38.13(6.44)$ & $36.12(8.42)$ \\
\hline Week 4 & $34.92(7.87)$ & $39.28(10.05)$ & $34.84(8.92)$ & $39.27(9.57)$ & $37.03(9.26)$ \\
\hline Week 9 & $35.91(7.96)$ & $39.91(9.40)$ & $38.48(9.85)$ & $40.52(9.06)$ & $38.66(9.12)$ \\
\hline Week 15 & $34.73(8.64)$ & $40.20(10.47)$ & $38.34(8.95)$ & $40.52(10.68)$ & $38.35(9.80)$ \\
\hline Change $^{\mathrm{a}}$ & $-2.89(7.69)$ & $-2.42(9.34)$ & $-1.14(4.79)$ & $-2.35(7.07)$ & $-2.20(7.40)$ \\
\hline
\end{tabular}

Mean (standard deviation) scores for pain, fatigue and function outcomes by treatment group and week are shown. No treatment group showed significant improvement over the others.

T/S, traditional needle locations with manual stimulation; T/0, traditional needle location without manual stimulation; N/S, nontraditional needle location with manual stimulation; N/0, nontraditional needle location without manual stimulation.

${ }^{a}$ Mean (SD) in change score (pain: week 0-13; fatigue and function: week 0-15).

${ }^{b}$ Model 1, $p$ value for comparison of groups: T/S + T/0 vs. N/S + N/0 (weeks 3, 8, and 13).

${ }^{c}$ Model 1, $p$ value for comparison of groups: T/S + N/S vs. T/0 + N/0 (weeks 3, 8, and 13). 
Table 3. Percentage of Responders by Acupuncture Group and Week

\begin{tabular}{|c|c|c|c|c|c|}
\hline & $T / S$ & $T / 0$ & $N / S$ & $N / 0$ & Total \\
\hline \multicolumn{6}{|l|}{ Pain } \\
\hline Week 3 & $8 / 29(28)$ & $6 / 30(20)$ & $5 / 28(18)$ & $6 / 27(22)$ & $25 / 114(22)$ \\
\hline Week 4 & $3 / 29(10)$ & $6 / 30(20)$ & $5 / 28(18)$ & $5 / 27(19)$ & $19 / 114$ (17) \\
\hline Week 8 & $6 / 29(21)$ & $10 / 30(33)$ & $10 / 28(36)$ & $6 / 27(22)$ & $32 / 114(28)$ \\
\hline Week 9 & $8 / 29(28)$ & $5 / 30(17)$ & $10 / 28(36)$ & $4 / 27(15)$ & $27 / 114(24)$ \\
\hline Week 13 & $8 / 29(28)$ & $13 / 30(43)$ & $9 / 28(32)$ & $7 / 27(26)$ & $37 / 114(32)$ \\
\hline Week 15 & $9 / 29(31)$ & $7 / 30(23)$ & $10 / 28(36)$ & $6 / 27(22)$ & $32 / 114(28)$ \\
\hline Change $^{\mathrm{a}}$ & 21 & 3 & 18 & 3 & 11 \\
\hline$p$, location ${ }^{\mathrm{b}}$ & 0.657 & & & & \\
\hline$p$, stimulation $^{\mathrm{c}}$ & 0.058 & & & & \\
\hline \multicolumn{6}{|l|}{ Fatigue } \\
\hline Week 4 & $3 / 29(10)$ & $5 / 30(17)$ & $3 / 28(11)$ & $2 / 27(7.4)$ & $13 / 114(11)$ \\
\hline Week 9 & $8 / 29(28)$ & $4 / 30(13)$ & $8 / 28(29)$ & $5 / 27$ (19) & $25 / 114(22)$ \\
\hline Week 15 & $4 / 29(14)$ & $6 / 30(20)$ & $9 / 28(32)$ & $4 / 27(15)$ & $23 / 114(20)$ \\
\hline Change $^{\mathrm{a}}$ & 4 & 3 & 21 & 7.6 & 9 \\
\hline \multicolumn{6}{|l|}{ Function } \\
\hline Week 4 & $7 / 29(24)$ & $5 / 30(17)$ & $1 / 28(3.6)$ & $3 / 27(11)$ & $16 / 114(14)$ \\
\hline Week 9 & $9 / 29(31)$ & $7 / 30(23)$ & $3 / 28(11)$ & $3 / 27$ (11) & $22 / 114(19)$ \\
\hline Week 15 & $6 / 29(21)$ & $8 / 30(27)$ & $1 / 28(3.6)$ & $4 / 27(15)$ & $19 / 114(17)$ \\
\hline Change $^{\mathrm{a}}$ & -3 & 10 & 0 & 4 & 3 \\
\hline
\end{tabular}

Number (percentage) of responders for each outcome by treatment group and week are shown. Clinically significant improvements in pain were observed in $25 \%-35 \%$ of subjects.

$\mathrm{T} / \mathrm{S}$, traditional needle location with manual stimulation; T/0, traditional needle location without manual stimulation; N/S, nontraditional needle location with manual stimulation; N/0, nontraditional needle location without manual stimulation.

aPercentage of responders changed between first and last assessment week (week 4 to week 15).

${ }^{b}$ Model 2, $p$ value for comparison of groups: T/S + T/0 vs. N/S + N/0 (weeks 3, 8, and 13).

${ }^{c}$ Model 2, $p$ value for comparison of groups: T/S + N/S vs. T/0 + N/0 (weeks 3, 8, and 13).

observed from either once to twice per week or from twice to three times per week (all $p>0.05$ ). No dose effect was observed within any of the treatment groups when analyzed separately (all $p>0.05$ ) possibly because of the smaller sample size per group compared to the entire cohort.

Figure 3B depicts the overall frequency dependence for NRS pain responders from all four groups combined. A trend of an increasing number of responders was observed and a noticeable decrease in analgesia was detected during the washout periods. This decrease in analgesia was significant from week 3 to $4(t=-2.787 ; p=0.007)$ and from weeks 13 and $15(t=-2.396 ; p=0.019)$. Together these data suggest that increasing treatment frequency decreased pain and that the cessation of treatment increased pain.

\section{Codependence of outcome domains}

To determine whether responder status was independent within the pain, fatigue, and function domains, multiple $2 \times$ $2 \chi^{2}$ tests of treatment responders were performed. Significant codependence in (1) pain-fatigue $\left(\mathrm{X}^{2}[1]=15.352 ; p=\right.$ $0.001)$, (2) fatigue-function $\left(\mathrm{X}^{2}[1]=10.469 ; p=0.001\right)$; and (3) pain-function $\left(\mathrm{X}^{2}[1]=10.045 ; p=0.002\right)$ was ob- served. These data suggest that responder status in all three outcome domains was highly interrelated.

\section{DISCUSSION}

Both randomized controlled trials, and longitudinal observation of individuals treated at tertiary care centers suggest that fibromyalgia is difficult to treat. ${ }^{25,26}$ As a result, no single intervention is completely accepted by the medical community, and no one intervention has significantly outperformed others. ${ }^{27,28}$ The present data provide evidence that a subset of individuals obtained improvements in clinical pain, fatigue, and function from acupuncture, although efficacy was not related to needle placement or stimulation. These results differed from Deluze et al., ${ }^{11}$ which may in part be caused by their use of electro-acupuncture, which is inherently difficult to blind and is a different treatment modality from the manual stimulation used here.

\section{Needle location and stimulation}

When performing sham acupuncture, investigators sometimes place needles on nontraditional acupuncture points using a technique termed "minimal acupuncture." 6 However 


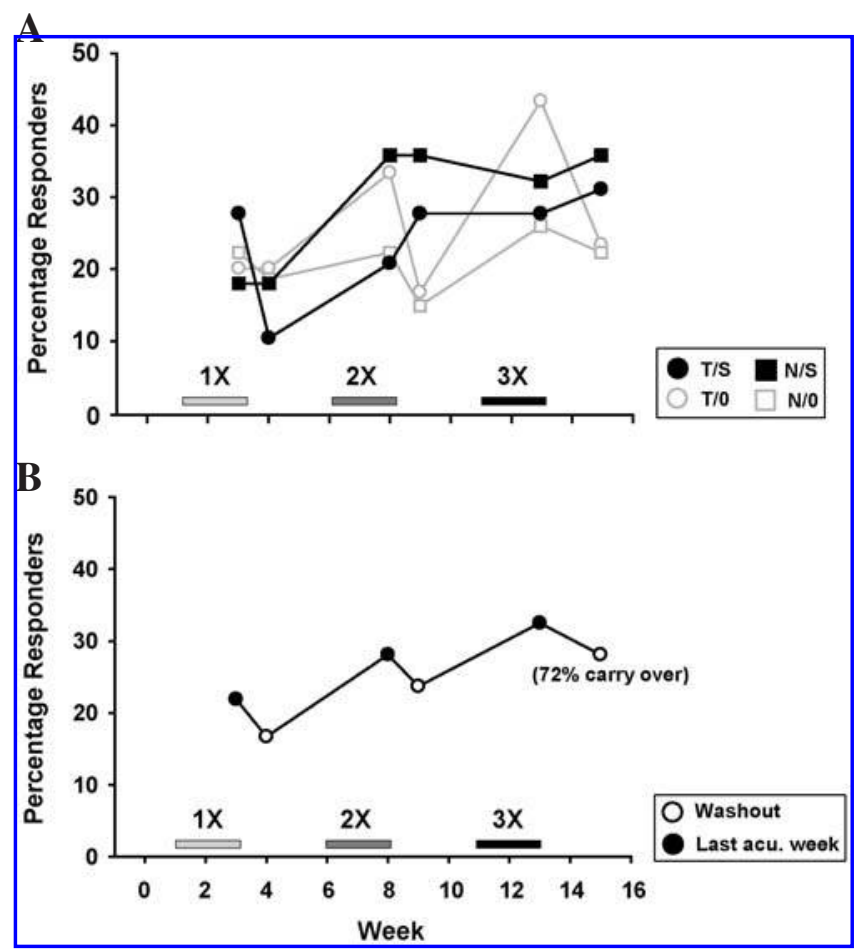

FIG. 3. Effects of needle location, stimulation, treatment frequency, and baseline depressive symptoms on pain responders. (A) Plot of percentage of NRS pain responders by group for increasing treatment frequency $(1 \times$ per week, $2 \times$ per week, and $3 \times$ per week). No one group consistently displayed more responders than another. (B) Plot of percentage NRS pain responders by increasing treatment frequency for all four groups combined. An increasing trend for responders was found. Solid symbols equal final week for each acupuncture dose (weeks 3, 8, and 13) while open symbols are assessment weeks (weeks 4, 9, and 15$)$. Seventy two percent $(72 \%)$ of responders after $3 \times$ per week were also responders after $2 \times$ per week. NRS, numeric rating scale. acu., acupuncture.

needle insertion, irrespective of location, may elicit a response that is functionally identical to that in traditional points. ${ }^{29-33}$ Indeed, sham needle sites have been shown to be more effective than inert controls, ${ }^{34}$ which implies that minimal acupuncture may not be inert. This may have been the case for the nontraditional groups, and perhaps the use of a more inert control intervention ${ }^{35,36}$ would clarify this issue.

Acupuncture advocates may suggest that FM is an inappropriate condition for the study of the specifics of needle placement. In Traditional Chinese Medicine, Ashi points are commonly used for the treatment of pain originating away from the major meridians, and needles are placed exactly where the pain is located, regardless of whether the pain lies on a meridian or does not correspond to a particular acupuncture point. ${ }^{1}$ Because FM pain is diffuse and located throughout the body, our nontraditional locations may have actually functioned as Ashi points.

Nonetheless no difference in clinical pain was detected regardless of whether the needles were stimulated. This in- dicates that the needle manipulation used in this study is not an essential component to acupuncture therapy in FM, and it follows that needle sensations elicited by manual manipulation are not critical in this setting. However it should be noted that some forms of acupuncture use no manual stimulation. The form of acupuncture administered here was highly simplified for experimental purposes; in a real clinical setting, elements such as needle stimulation might enhance the effectiveness of this therapy.

\section{Frequency dependence}

A hallmark of effective pharmacologic interventions is the dose-response effect. In theory, interventions that effect changes in physiological substrates should result in dose dependency, in which a greater effect is elicited with an increase in the magnitude of the therapeutic intervention. In exploratory analyses, data were reported supporting this hypothesis for an overall effect of treatment frequency in FM. This effect was modest, as it was only observed when all four treatment groups were combined. With the absence of a no-treatment arm, one cannot exclude regression to the mean, natural history, or other time effects as possible mechanisms for the frequency dependence because time and dose were confounded in this study. However this seems unlikely for two reasons: first, an overall decay in analgesia was observed during the wash-out periods (Fig. 3B), suggesting that treatment was related to pain relief; and second, the natural trend of pain fluctuation in fibromyalgia does not match the magnitudes observed here. ${ }^{25}$

\section{Reduction of pain in fibromyalgia by a "placebo effect" of acupuncture}

In light of the fact that no differences were observed for either stimulation or location of the needles within this study, one may be tempted to conclude that the effects of acupuncture occur largely because of a "placebo effect." Indeed the criteria used to designate responders from nonresponders lies well within the range of the placebo effect. ${ }^{37}$ The placebo effect may have diluted any specific effects of acupuncture observed in this trial; however further studies will be required to confirm or refute these findings.

\section{CONCLUSIONS}

In this controlled trial, a subset of FM subjects received symptom improvement with acupuncture needle insertion, but no specific effects of needle placement or stimulation were noted. Alternative designs containing more "inert" comparison groups or trials with fewer dropout rates may be more helpful in assessing which specific factors are necessary to engender these effects. More specialized treatment regimens tailored to individual FM participants may also yield more positive findings. 


\section{ACKNOWLEDGMENTS}

The authors thank Jack Kalbfleisch, John Pezzullo, and Cora MacPherson for their statistical advice and Dedra Buchwald for careful reading of the manuscript. This project was supported by the National Institutes of Health (NCCAM) grant R01 AT00004, the Department of Defense DAMD-17002-0018, and Georgetown University GCRC grant 98-186. R.E.H. was supported by National Institutes of Health (NCCAM) K01 AT01111-01.

\section{REFERENCES}

1. Xinnong C. Chinese Acupuncture and Moxibustion. Bejing: Foreign Languages Press, 1999.

2. Eisenberg DM, Davis RB, Ettner SL, et al. Trends in alternative medicine use in the United States, 1990-1997: Results of a follow-up national survey. JAMA 1998;280:15691575.

3. Emslie MJ, Campbell MK, Walker KA. Changes in public awareness of, attitudes to, and use of complementary therapy in North East Scotland: Surveys in 1993 and 1999. Complement Ther Med 2002;10:148-153.

4. NIH Consensus Conference. Acupuncture. JAMA 1998;280: $1518-1524$.

5. Streitberger K, Vickers A. Placebo in acupuncture trials. Pain 2004;109:195-199.

6. Stux G, Hammerschlag R. Clinical Acupuncture: Scientific Basis. New York: Springer, 2001.

7. Ballegaard S, Pedersen F, Pietersen A, et al. Effects of acupuncture in moderate, stable angina pectoris: A controlled study. J Intern Med 1990;227:25-30.

8. Tavola T, Gala C, Conte G, Invernizzi G. Traditional Chinese acupuncture in tension-type headache: a controlled study. Pain 1992;48:325-329.

9. Takeda W, Wessel J. Acupuncture for the treatment of pain of osteoarthritic knees. Arthritis Care Res 1994;7:118-122.

10. Thomas M, Eriksson SV, Lundeberg T. A comparative study of diazepam and acupuncture in patients with osteoarthritis pain: A placebo controlled study. Am J Chin Med 1991; 19:95-100.

11. Deluze C, Bosia L, Zirbs A, et al. Electroacupuncture in fibromyalgia: results of a controlled trial. BMJ 1992;305: $1249-1252$.

12. Wolfe F, Ross K, Anderson J, et al. The prevalence and characteristics of fibromyalgia in the general population. Arthritis Rheum 1995;38:19-28.

13. Jacobsen S, Bredkjaer SR. The prevalence of fibromyalgia and widespread chronic musculoskeletal pain in the general population. Scand J Rheumatol 1992;21:261-263.

14. Wolfe F, Smythe HA, Yunus MB, et al. The American College of Rheumatology 1990 Criteria for the Classification of Fibromyalgia. Report of the Multicenter Criteria Committee. Arthritis Rheum 1990;33:160-172.

15. Granges G, Littlejohn G. Pressure pain threshold in pain-free subjects, in patients with chronic regional pain syndromes, and in patients with fibromyalgia syndrome. Arthritis Rheum 1993;36:642-646.

16. Yunus MB. Towards a model of pathophysiology of fibromyalgia: Aberrant central pain mechanisms with peripheral modulation. J Rheumatol 1992;19:846-850.

17. Berman BM, Swyers JP. Complementary medicine treatments for fibromyalgia syndrome. Baillieres Best Pract Res Clin Rheumatol 1999;13:487-492.

18. Waylonis GW. Long-term follow-up on patients with fibrositis treated with acupuncture. Ohio State Med J 1977; 73:299-302.

19. Lautenschlager J, Schnorrenberger CC, Muller W. Dtsch Zschr Akup 1989;6:122-128.

20. Berman BM, Ezzo J, Hadhazy V, Swyers JP. Is acupuncture effective in the treatment of fibromyalgia? J Fam Pract 1999;48:213-218.

21. Farrar JT, Young JP Jr, LaMoreaux L, et al. Clinical importance of changes in chronic pain intensity measured on an 11point numerical pain rating scale. Pain 2001;94:149-158.

22. Smets EM, Garssen B, Bonke B, De Haes JC. The Multidimensional Fatigue Inventory (MFI) psychometric qualities of an instrument to assess fatigue. J Psychosom Res 1995; 39:315-325.

23. Jacobson NS, Truax P. Clinical significance: A statistical approach to defining meaningful change in psychotherapy research. J Consult Clin Psychol 1991;59:12-19.

24. Ware JEJ, Snow K, Kosinski M, Gandek B. SF-36 Health Survey Manual and Interprestation Guide. Lincoln, RI: QualityMetric, Inc., 2000.

25. Wolfe F, Anderson J, Harkness D, et al. Health status and disease severity in fibromyalgia: Results of a six- center longitudinal study [see comments]. Arthritis Rheum 1997;40:1571-1579.

26. Fitzcharles MA, Costa DD, Poyhia R. A study of standard care in fibromyalgia syndrome: A favorable outcome. J Rheumatol 2003;30:154-159.

27. Crofford LJ, Appleton BE. The treatment of fibromyalgia: A review of clinical trials. Curr Rheumatol Rep 2000;2: $101-103$

28. Rossy LA, Buckelew SP, Dorr N, et al. A meta-analysis of fibromyalgia treatment interventions. Ann Behav Med 1999; 21:180-191.

29. Leibing E, Leonhardt U, Koster G, et al. Acupuncture treatment of chronic low-back pain-a randomized, blinded, placebo-controlled trial with 9-month follow-up. Pain 2002; 96:189-196.

30. Goddard G, Karibe H, McNeill C, Villafuerte E. Acupuncture and sham acupuncture reduce muscle pain in myofascial pain patients. J Orofac Pain 2002;16:71-76.

31. Ernst E. Acupuncture as a symptomatic treatment of osteoarthritis. A systematic review. Scand J Rheumatol 1997;26: 444-447.

32. Shlay JC, Chaloner K, Max MB, et al. Acupuncture and amitriptyline for pain due to HIV-related peripheral neuropathy: A randomized controlled trial. Terry Beirn Community Programs for Clinical Research on AIDS. JAMA 1998;280: 1590-1595.

33. Margolin A, Kleber HD, Avants SK, et al. Acupuncture for the treatment of cocaine addiction: A randomized controlled trial. JAMA 2002;287:55-63. 
34. Fargas-Babjak A. Acupuncture, transcutaneous electrical nerve stimulation, and laser therapy in chronic pain. Clin J Pain 2001;17:S105-S113.

35. Streitberger K, Kleinhenz J. Introducing a placebo needle into acupuncture research. Lancet 1998;352:364-365.

36. Sherman KJ, Hogeboom CJ, Cherkin DC, Deyo RA. Description and validation of a noninvasive placebo acupuncture procedure. J Altern Complement Med 2002;8:11-19.

37. Turner JA, Deyo RA, Loeser JD, et al. The importance of placebo effects in pain treatment and research. JAMA 1994;271:1609-1614.
Address reprint requests to: Richard E. Harris, Ph.D. Department of Internal Medicine Division of Rheumatology University of Michigan Chronic Pain and Fatigue Research Center 24 Frank Lloyd Wright Drive, P.O. Box 385 Ann Arbor, MI 48106

E-mail: reharris@med.umich.edu 


\section{This article has been cited by:}

1. Howard H. Moffet . 2009. Sham Acupuncture May Be as Efficacious as True Acupuncture: A Systematic Review of Clinical TrialsSham Acupuncture May Be as Efficacious as True Acupuncture: A Systematic Review of Clinical Trials. The Journal of Alternative and Complementary Medicine 15:3, 213-216. [Abstract] [PDF] [PDF Plus]

2. Vitaly Napadow, Andrew Ahn, John Longhurst, Lixing Lao, Elisabet Stener-Victorin , Richard Harris, Helene M. Langevin . 2008. The Status and Future of Acupuncture Clinical ResearchThe Status and Future of Acupuncture Clinical Research. The Journal of Alternative and Complementary Medicine 14:7, 861-869. [Abstract] [PDF] [PDF Plus]

3. Roland Staud. 2008. Mechanisms of acupuncture analgesia: Effective therapy for musculoskeletal pain?. Current Rheumatology Reports 9:6, 473-481. [CrossRef]

4. Michael Francis Johnston, Bo Xiao, Ka-Kit Hui. 2007. Acupuncture and fatigue: Current basis for shared communication between breast cancer survivors and providers. Journal of Cancer Survivorship 1:4, 306-312. [CrossRef]

5. Roland Staud. 2007. Treatment of fibromyalgia and its symptoms. Expert Opinion on Pharmacotherapy 8:11, 1629-1642. [CrossRef]

6. Daniel S Rooks. 2007. Fibromyalgia treatment update. Current Opinion in Internal Medicine 6:3, 328-334. [CrossRef]

7. Hélène M. Langevin , Richard Hammerschlag, Lixing Lao , Vitaly Napadow , Rosa N. Schnyer , Karen J. Sherman . 2006. Controversies In Acupuncture Research: Selection of Controls and Outcome Measures In Acupuncture Clinical TrialsControversies In Acupuncture Research: Selection of Controls and Outcome Measures In Acupuncture Clinical Trials. The Journal of Alternative and Complementary Medicine 12:10, 943-953. [Citation] [PDF] [PDF Plus]

8. Dina Dadabhoy, Daniel J Clauw. 2006. Therapy Insight: fibromyalgia-a different type of pain needing a different type of treatment. Nature Clinical Practice Rheumatology 2:7, 364-372. [CrossRef]

9. Roland Staud, Donald D Price. 2006. Mechanisms of acupuncture analgesia for clinical and experimental pain. Expert Review of Neurotherapeutics 6:5, 661-667. [CrossRef]

10. Daniel J Clauw. 2006. Does acupuncture help reduce pain in patients with fibromyalgia?. Nature Clinical Practice Rheumatology $1: 2,76-77$. [CrossRef]

11. 2005. Literature WatchLiterature Watch. Alternative and Complementary Therapies 11:5, 273-274. [Citation] [PDF] [PDF Plus] 